Short communication

\title{
Development and characterization of microsatellite markers for Osyris lanceolata Hochst. \& Steud., an endangered African sandalwood tree species
}

\author{
John O. Otieno ${ }^{1,3} *$, Stephen F. Omondi ${ }^{1}$, Annika Perry ${ }^{2}$, David W. Odee ${ }^{1,2}$, \\ Emmanuel T. Makatiani ${ }^{1}$, Oliver Kiplagat ${ }^{3}$ and Stephen Cavers ${ }^{2}$ \\ ${ }^{1}$ Kenya Forestry Research Institute, P.O. Box 20412-00200, Nairobi Kenya \\ ${ }^{2}$ Centre for Ecology and Hydrology, CEH Edinburgh, Bush Estate Penicuik, Midlothian, United Kingdom \\ ${ }^{3}$ University of Eldoret, P.O. Box 1125-30100, Eldoret, Kenya
}

*Corresponding Author: jochiengo@gmail.com

[Accepted: 28 December 2016]

[Cite as: Otieno JO, Omondi SF, Perry A, Odee DW, Makatiani ET, Kiplagat O \& Cavers S (2016) Development and characterization of microsatellite markers for Osyris lanceolata Hochst. \& Steud., an endangered African sandalwood tree species. Tropical Plant Research 3(3): 701-703]

Osyris lanceolata Hochst. \& Steud. is a multipurpose tree species widely spread in many of the sub-Saharan countries ranging from Algeria to Ethiopia all the way to South Africa. In Kenya, the species is endemic to the Arid and Semi-Arid Lands (ASALs). It is highly valued for its essential oils used in the cosmetic and pharmaceutical industries. Despite its endangered status and economic importance, little is known about its genetic diversity status and only few conservation strategies exist for the species. Overexploitation of the species has resulted in the decline of its population and reduced availability of its products. The mode of harvesting of sandalwood is destructive and unsustainable. This is because the whole tree is usually uprooted to get the heartwood from the stem, stump and roots. The exploitation of African sandalwood could soon drive the species to extinction unless proper control measures are put in place through regulation of its trade and development of conservation strategies. Despite its endangered status and economic importance, no genetic study has been carried out on the species to provide information vital for conservation strategies. This paper reports the development and characterization of a set of 12 polymorphic and five (5) monomorphic microsatellite markers isolated and characterized of $O$. lanceolata.

One plant leaf sample was used as the source of DNA for genomic library construction. Total genomic DNA was extracted from silica gel dried leaf using DNeasy Plant Mini Kit (QIAGEN, Hilden, Germany). The DNA sample was then sent to The Gene Pool Institute of Evolutionary Biology, University of Edinburgh for sequencing. Simple sequence repeats (SSRs) were extracted through PAL Finder software version 0.02.04 (Castoe et al. 2012) and primer pairs developed. Identified microsatellites and designed primers were assembled using QDD (Meglécz et al. 2010) with parameters given in set_qdd_default.ini.file. The gaps emerging during the scaffolding process were closed using GapCloser (vs. 1.12). The contigs >1000 bp of the draft assembly were analyzed and functionally annotated using Blast2GO (Conesa et al. 2005). Based on this information, 48 primer pairs consisting of either di- or trinucleotide repeats were selected. After testing, 17 primer pairs were identified and used to characterize 84 samples of $O$. lanceolata from three natural populations, namely Mt. Elgon (28), Gachuthi (27) and Kitui (29). The PCR analysis was performed using Multiplex PCR Mater Mix (QIAGEN) and $10 \mathrm{ng}$ of DNA as described by (Omondi et al. 2015). The PCR mix contained a fluorescently labelled M13 primer, M13-tailed forward primer and a reverse primer in the concentration ratio of 0.15:0.01:0.15 $\mu \mathrm{M}$. For all loci, a touchdown thermal cycling program was used with annealing temperature ranging between $57-55^{\circ} \mathrm{C}$. The cycling profile consisted of initial denaturation of $95^{\circ} \mathrm{C}$ for $15 \mathrm{~min}$ followed by 10 cycles at $94^{\circ} \mathrm{C}$ for $30 \mathrm{~s}, 57^{\circ} \mathrm{C}$ for $90 \mathrm{~s}$ and $72^{\circ} \mathrm{C}$ for $60 \mathrm{~s}$ (annealing temperature decreasing by $1^{\circ} \mathrm{C}$ per cycle); and 22 cycles at $94^{\circ} \mathrm{C}$ for $30 \mathrm{~s}, 55^{\circ} \mathrm{C}$ for $90 \mathrm{~s}$ and $72^{\circ} \mathrm{C}$ for $60 \mathrm{~s}$ and a single final cycle at $60^{\circ} \mathrm{C}$ for 30 min using Verity 96 well thermocycler (Applied Biosystems). 
Otieno et al. (2016) 3(3): 701-703

Table 1. Descriptive statistics over all loci for the three natural populations of Osyris lanceolata Hochst. \& Steud.

\begin{tabular}{|c|c|c|c|c|c|c|c|c|c|c|c|}
\hline & & & & & & $\mathbf{H}_{\mathrm{O}}$ & $\mathbf{H}_{\mathrm{O}}$ & $\mathrm{H}_{\mathrm{O}}$ & $\mathbf{H}_{\mathrm{E}}$ & $\mathbf{H}_{\mathrm{E}}$ & $\mathbf{H}_{\mathrm{E}}$ \\
\hline $\begin{array}{l}\text { DDBJ } \\
\text { GenBank } \\
\text { accession no. }\end{array}$ & Locus & Primer sequences $\left(5^{\prime}-3^{\prime}\right)$ & $\begin{array}{c}\text { Repeat } \\
\text { motif }\end{array}$ & $\begin{array}{l}\text { size } \\
\text { range } \\
(\text { bp) }\end{array}$ & $\mathbf{N a}$ & $\begin{array}{l}\text { Mt. } \\
\text { Elgon }\end{array}$ & Gachuthi & Kitui & $\begin{array}{l}\text { Mt. } \\
\text { Elgon }\end{array}$ & Gachuthi & Kitui \\
\hline LC126834 & KFOL2 & $\begin{array}{l}\text { F:AGAATGTCATTTGAAGGCTCGA } \\
\text { R:CCTTTCCTCCGTTCTCCTCG }\end{array}$ & CGTC & $178-194$ & 5 & 0.393 & 0.556 & 0.483 & 0.572 & 0.626 & 0.569 \\
\hline LC154965 & KFOL7 & $\begin{array}{l}\text { F: CTGTGCAATGGAGAAGGCCA } \\
\text { R:CGCGGGATTGGGATGTCATA }\end{array}$ & ATT & $115-120$ & 2 & 0.043 & 0.000 & 0.000 & 0.043 & 0.000 & 0.000 \\
\hline LC154966 & KFOL8 & $\begin{array}{l}\text { F:GCTGCTTCTACGGTCACTGT } \\
\text { R:GTGGTGGATATGGAGGTGGC }\end{array}$ & CCG & $120-130$ & 2 & 0.000 & 0.200 & 0.462 & 0.073 & 0.184 & 0.434 \\
\hline LC126835 & KFOL13 & $\begin{array}{l}\text { F:TCCGAGGAACAGGGACTCTT } \\
\text { R:AGCGAAGAACTCATGAGCGAA }\end{array}$ & $\mathrm{AC}$ & $139-165$ & 7 & 0.556 & 0.148 & 0.069 & 0.552 & 0.139 & 0.067 \\
\hline LC154967 & KFOL15 & $\begin{array}{l}\text { F:CATTGACGAATTGCATCCCGT } \\
\text { R:CGTGAAGTTCAGTGCAAACC }\end{array}$ & CGC & $145-150$ & 2 & 0.000 & 0.000 & 0.000 & 0.000 & 0.000 & 0.000 \\
\hline LC154968 & KFOL16 & $\begin{array}{l}\text { F:TGGAGCCCATTCTCTTTCCTT } \\
\text { R:TGCACGTATTCCACATTTCCA }\end{array}$ & GT & $130-160$ & 5 & 0.107 & 0.333 & 0.107 & 0.103 & 0.352 & 0.166 \\
\hline LC126836 & KFOL17 & $\begin{array}{l}\text { F:CATTGACGAATTGCATCCCGT } \\
\text { R:CGTGAAGTTCAGTGCAAACC }\end{array}$ & AG & $178-220$ & 21 & 0.893 & 0.741 & 0.793 & 0.879 & 0.824 & 0.863 \\
\hline LC154969 & KFOL19 & $\begin{array}{l}\text { F:GGTAGCGAGCGGTGATATGT } \\
\text { R:ACCTAACAACTTGAAGCTCTCCC }\end{array}$ & TC & $200-230$ & 3 & 0.259 & 0.000 & 0.000 & .338 & 0.000 & .000 \\
\hline LC126838 & KFOL24 & $\begin{array}{l}\text { F:CAACTCGATCGTGCATTGGC } \\
\text { R:TCCGCATATCCATTTGGCCG }\end{array}$ & CT & $219-263$ & 15 & 0.821 & 0.192 & 0.276 & 0.902 & 0.286 & 0.452 \\
\hline LC154970 & KFOL27 & $\begin{array}{l}\text { F:CTAAACTGTCAGGGCTTGCT } \\
\text { R:ATACCTTAGCTCCCGTTGCG }\end{array}$ & ATG & $225-230$ & 1 & 0.000 & 0.000 & 0.000 & 0.000 & 0.000 & 0.000 \\
\hline LC126839 & KFOL28 & $\begin{array}{l}\text { F:ATAAAGGCCCACGAGCTCAG } \\
\text { R:AACATCGCCATGCAGAACAG }\end{array}$ & CT & $245-255$ & 5 & 0.714 & 0.000 & 0.069 & 0.605 & 0.000 & 0.067 \\
\hline LC154961 & KFOL29 & $\begin{array}{l}\text { F:GCTGAATCAGGGACAGGCAT } \\
\text { R:GGCCTCGAACAAAGTGCATG }\end{array}$ & GA & $230-250$ & 2 & 0.000 & 0.074 & 0.034 & 0.000 & 0.073 & 0.034 \\
\hline LC126840 & KFOL30 & $\begin{array}{l}\text { F:CTAAACTGTCAGGGCTTGCT } \\
\text { R:ATACCTTAGCTCCCGTTGCG }\end{array}$ & $\mathrm{TC}$ & 270-306 & 12 & 0.643 & 0.333 & 0.483 & 0.614 & 0.471 & 0.663 \\
\hline LC126841 & KFOL37 & $\begin{array}{l}\text { F:TTTCTAGAGCTAACATACCTCTGAA } \\
\text { R:ATGACCTGGGTGCTTTGCTG }\end{array}$ & TG & $300-340$ & 17 & 0.889 & 0.185 & 0.517 & 0.853 & 0.278 & 0.609 \\
\hline LC126843 & KFOL42 & $\begin{array}{l}\text { F:AGGTCCTCCTGCCTGAGAAT } \\
\text { R: CATAGGGCTGTGATGCGTCA }\end{array}$ & TG & $315-337$ & 6 & 0.308 & 0.037 & 0.000 & 0.277 & 0.036 & 0.000 \\
\hline LC126844 & KFOL47 & $\begin{array}{l}\text { F:TTTGATCGTAAATTATAGATGTCCACA } \\
\text { R:CCCTTGCTTGATCTCCAGGTA }\end{array}$ & $\mathrm{CA}$ & $353-387$ & 15 & $0.393^{*}$ & 0.731 & 0.759 & 0.791 & 0.771 & 0.826 \\
\hline LC126845 & KFOL48 & $\begin{array}{l}\text { F:GAGTGCATGGAATTATGTGCGT } \\
\text { R:TCGCCATGAGAAGGGTTACT }\end{array}$ & $\mathrm{TC}$ & $369-393$ & 12 & 0.357 & 0.519 & 0.621 & 0.343 & 0.666 & 0.519 \\
\hline
\end{tabular}

Note: 5 M13 tail: TGTAAAACGACGGCCAGT; F, forward sequence; R, reverse sequence; Na, number of observed alleles per locus, $\mathrm{H}_{\mathrm{O}}$ heterozygosity observed with $P$-values for the Hardy- Weinberg equilibrium test and significance threshold adjusted using the Bonferroni correction: $* P<0.05, \mathrm{H}_{\mathrm{E}}$ heterozygosity expected.

Amplified fragments were analyzed against an internal standard (Liz 600 size standard) on an ABI 3500 (Applied Biosystems). Alleles were visualized and scored using GeneMapper version 5 (Applied Biosystems). The genetic parameters were determined using GenAlex software v 6.4 (Peakall \& Smouse 2012). Deviations from Hardy-Weinberg equilibrium (HWE) and linkage disequilibrium (LD) was determined using Genepop online software version (http://wbiomed.curtin.edu.au/genepop/).

The number of alleles per locus across the three populations ranged from one (KFOL27) to 21 (KFOL17). Expected heterozygosity ranged from 0.00 (KFOL15, KFOL27 and KFOL29) to 0.902 (KFOL24) in Mt. Elgon population, from 0.00 (KFOL28) to 0.824 (KFOL7, KFOL15, KFOL17, KFOL19 and KFOL27) in Gachuthi population, 0.00 (KFOL7, KFOL15, KFOL19, KFOL27 and KFOL42) to 0.863 (KFOL17) in Kitui population (Table 1). Total paternity exclusion probability (Pe) over all loci was 0.989 . Only one pair of loci (KFOL16 KFOL37) showed significant LD at the $5 \%$ level after Bonferroni correction. Deviation from HWE was detected for one locus (KFOL47) in Mt. Elgon population (Table 1). Out of the 17 markers developed, 12 were polymorphic while five (KFOL7, KFOL8, KFOL15, KFOL27 and KFOL29) were monomorphic.

The 17 microsatellite markers developed are the first reported for $O$. lanceolata and are suitable for population genetic studies due to their high polymorphic characteristics. The markers will be used for studying genetic diversity and population structure across the distribution range, and to assess levels of gene flow 
between populations. These studies will be important in designing sustainable management and conservation strategies for the species.

\section{ACKNOWLEDGMENTS}

This study was funded and conducted by collaboration between Kenya Forestry Research Institute (KEFRI) and Centre of Ecology and Hydrology (CEH) of UK, under International Timber Tracking Organization (ITTO) project contract No.: 2005-34135-16007. We wish to thank Mr. John Gicheru and Mr. Charles Oduor of KEFRI for helping in sample collection and laboratory work.

\section{REFERENCES}

Castoe TA, Poole AW, de Koning APJ, Jones KL, Tomback DF, Oyler-McCance SJ, Fike JA, Lance SL, Streicher JW, Smith EN \& Pollock DD (2012) Rapid Microsatellite Identification from Illumina paired-end genomic sequencing in two birds and a snake. PLoS ONE 7: e30953.

Conesa A, Götz AS, Garcia-Gomez JM, Terol J, Talon JM \& Robles M. (2005) Blast2GO: a universal tool for annotation, visualization and analysis in functional genomics research. Bioinformatics 21: 3674-3676.

Meglécz E, Costedoat C, Dubut V, Gilles A, Malausa T, Pech N \& Martin JF (2010) QDD: a user-friendly program to select microsatellite markers and design primers from large sequencing projects. Bioinformatics 26: 403-404.

Omondi SF, Machua J, Gicheru JM \&, So Hanaoka (2015). Isolation and characterization of microsatellite markers for Acacia tortilis (Forsk.) Hayne. Conservation Genetics Resources 7 (2): 529-531.

Peakall R \& Smouse PE (2012) GenAlEx 6.5: genetic analysis in Excel. Population genetic software for teaching and research-an update. Bioinformatics 28: 2537-2539. 